PROCEEDINGS OF THE

AMERICAN MATHEMATICAL SOCIETY

Volume 137, Number 1, January 2009, Pages 99-106

S 0002-9939(08)09496-3

Article electronically published on July 10, 2008

\title{
NIVEAU SPECTRAL SEQUENCES ON SINGULAR SCHEMES AND FAILURE OF GENERALIZED GERSTEN CONJECTURE
}

\author{
PAUL BALMER
}

(Communicated by Paul Goerss)

\begin{abstract}
We construct a new local-global spectral sequence for Thomason's non-connective $K$-theory, generalizing the Quillen spectral sequence to possibly non-regular schemes. Our spectral sequence starts at the $E_{1}$-page where it displays Gersten-type complexes. It agrees with Thomason's hypercohomology spectral sequence exactly when these Gersten-type complexes are locally exact, a condition which fails for general singular schemes, as we indicate.
\end{abstract}

Our main result is the following application of abstract triangular geometry [2].

Theorem 1. Let $X$ be a (topologically) noetherian scheme of finite Krull dimension. Then there exists a spectral sequence whose first page is

$$
E_{1}^{p, q}=\bigoplus_{x \in X^{(p)}} K_{-p-q}\left(\mathcal{O}_{X, x} \text { on }\{x\}\right) \quad \text { for } p, q \in \mathbb{Z},
$$

converging toward $K_{-n}(X)$ for $n \in \mathbb{Z}$, "along $n=p+q$ "; that is, the indexing in the spectral sequence is such that $d_{r}: E_{r}^{p, q} \rightarrow E_{r}^{p+r, q-r+1}$ for $r \geq 1$. Here, $K_{*}(X$ on $Y)$ stands for Thomason's non-connective (or "Bass") $K$-theory of those perfect complexes of $\mathcal{O}_{X}$-modules which are acyclic on $X-Y$; see [12, §6].

Note the presence of negative $K$-groups, a crucial fact throughout the paper. Negative $K$-theory roots back to work of Bass and of Karoubi, independently.

For $X$ regular, this coniveau spectral sequence is due to Quillen [10, Thm. 5.4], who also used dévissage to replace the local terms $K_{*}\left(\mathcal{O}_{X, x}\right.$ on $\left.\{x\}\right)$ by $K$-groups of residue fields, $K_{*}(\kappa(x))$. Although Theorem 1 can also be proved by starting with (10.3.6) in Thomason [12, proof of Thm. 10.3], the conceptual proof given here relies on deep geometric facts and easily transposes to other theories; see Remark 3 Important progress appeared even before [12, e.g. in Levine [8] or Weibel [15, 16, 17, but always under restrictions on the singularities. The above theorem seems to provide the most general coniveau spectral sequence one could wish for. Our proof is a direct application of two recent results : first, Schlichting's localization long exact sequence involving negative $K$-groups (see [11]), and second, the author's decomposition in local terms of the idempotent completion of the successive quotients of the coniveau filtration of $D^{\text {perf }}(X)$; see [2] or (7) below. These two recent papers,

Received by the editors September 17, 2007, and, in revised form, January 9, 2008.

2000 Mathematics Subject Classification. Primary 19E08, 19D35, 18E30.

Key words and phrases. Spectral sequence, $K$-theory, singular schemes.

The author's research was supported by NSF grant 0654397. 
2] and [11, grew as new branches of the same trunk, namely Thomason's masterpiece [12 mentioned above. Actually, in [12, Thm. 10.3], Thomason constructs another spectral sequence, the hypercohomology spectral sequence

$$
E_{2}^{p, q}=H_{\text {Zar }}^{p}\left(X, \mathcal{K}_{-q}\right) \stackrel{p+q=n}{\Longrightarrow} K_{-n}(X),
$$

where $\mathcal{K}_{m}$ is the sheafification of the presheaf $U \mapsto K_{m}(U)$, for $m \in \mathbb{Z}$. It is legitimate to ask whether the $E_{2}$-page of our spectral sequence (10) coincides with that of (2). We shall see in Remark 6 that this fails in general. Note that our spectral sequence (11) is potentially interesting even for local schemes, where the vanishing of cohomology forces Thomason's spectral sequence (2) to be trivial.

Actually, we can also construct a niveau spectral sequence, involving dimension instead of codimension. To synthesize, let us consider a general dimension function

$$
\operatorname{dim}: \operatorname{Irred}(X) \longrightarrow \mathbb{Z}
$$

on the set $\operatorname{Irred}(X)$ of irreducible closed subsets of $X$; i.e., we assume $\operatorname{dim}(Y)<$ $\operatorname{dim}\left(Y^{\prime}\right)$ for every two $Y, Y^{\prime} \in \operatorname{Irred}(X)$ such that $Y \subset Y^{\prime}$ and $Y \neq Y^{\prime}$. The Krull dimension $\operatorname{dim}(Y):=\operatorname{dim}_{\mathrm{Krull}}(Y)$ is an example, and so is the opposite of the Krull codimension $\operatorname{dim}(Y):=-\operatorname{codim}_{\mathrm{Krull}}(Y)$, but there are other dimension functions, e.g. in intersection theory. As usual, we extend our dimension function to any nonempty closed $Z \subset X$ by $\operatorname{dim}(Z):=\max \{\operatorname{dim}(Y) \mid Y \in \operatorname{Irred}(X)$ and $Y \subset Z\} \in \mathbb{Z}$ (and $\operatorname{dim}(\emptyset):=-\infty)$. We moreover assume that there exists $d \in \mathbb{N}$ such that

$$
-d \leq \operatorname{dim}(Z) \leq d
$$

for every non-empty closed subset $Z \subset X$. This obviously holds for dim Krull and for - codim $_{\text {Krull }}$ since we assume $X$ to have finite Krull dimension.

For every $i \in \mathbb{Z}$, we define $X_{(i)}:=\{x \in X \mid \operatorname{dim}(\overline{\{x\}})=i\}$. Note that the set $X^{(i)}=\left\{x \in X \mid \operatorname{dim}_{\text {Krull }}\left(\mathcal{O}_{X, x}\right)=i\right\}$ of Theorem 1 is nothing but $X_{(-i)}$ for the dimension function - codim $_{\text {Krull }}$. With this general notion of dimension, we prove:

Theorem 2. Let $X$ be a topologically noetherian scheme with a dimension function (3) satisfying (4). Then there exists a converging spectral sequence

$$
E_{1}^{p, q}=\bigoplus_{x \in X_{(-p)}} K_{-p-q}\left(\mathcal{O}_{X, x} \text { on }\{x\}\right) \stackrel{p+q=n}{\Longrightarrow} K_{-n}(X) .
$$

Proof. We define for each $p \in \mathbb{Z}$ the following subcategory of $\mathrm{D}^{\text {perf }}(X)$ :

$$
\mathrm{D}_{p}=\mathrm{D}_{(\leq p)}^{\text {perf }}(X):=\left\{E \in \mathrm{D}^{\text {perf }}(X) \mid \operatorname{dim}(\operatorname{supph}(E)) \leq p\right\},
$$

where the closed subset $\operatorname{supph}(E) \subset X$ is the support of the total homology of the perfect complex $E$. Because of (4), we have a finite filtration

$$
0=\mathrm{D}_{-d-1} \subset \mathrm{D}_{-d} \subset \cdots \subset \mathrm{D}_{p-1} \subset \mathrm{D}_{p} \subset \cdots \subset \mathrm{D}_{d}=\mathrm{D}^{\text {perf }}(X) .
$$

Since this filtration can be realized at the level of Waldhausen models, there is a "naive" spectral sequence starting with the Waldhausen $K$-theory of (some model of) $\mathrm{D}_{p} / \mathrm{D}_{p-1}$ and converging to Thomason's connective $K$-theory of $X$. This would lead to Quillen's classical spectral sequence for $X$ regular. Nevertheless there is no good description of the quotient $\mathrm{D}_{p} / \mathrm{D}_{p-1}$ when $X$ is singular. Still, following Thomason's insight of [12, $\S 5]$, we can instead describe the idempotent completion of this quotient. Recall from $[3$ that the idempotent completion $\widetilde{\mathcal{K}}$ of a triangulated category $\mathcal{K}$ inherits a unique triangulation such that $\mathcal{K} \hookrightarrow \widetilde{\mathcal{K}}$ is exact. Then, 
by [2, Thm. 3.24 and $\S 4.1]$, we know that for every $p \in \mathbb{Z}$, localization induces an equivalence

$$
\widetilde{\mathrm{D}_{p} / \mathrm{D}_{p-1}} \stackrel{\sim}{\longrightarrow} \coprod_{x \in X_{(p)}} \mathrm{D}_{\{x\}}^{\text {perf }}\left(\mathcal{O}_{X, x}\right)
$$

between the idempotent completion of the quotient $\mathrm{D}_{p} / \mathrm{D}_{p-1}$ and the coproduct over $x \in X_{(p)}$ of the derived categories of perfect complexes of $\mathcal{O}_{X, x}$-modules with homology supported on the closed point $x \in \operatorname{Spec}\left(\mathcal{O}_{X, x}\right)$.

The $K$-theoretic price to pay for introducing idempotent completions is the appearance of negative $K$-groups in the localization long exact sequence. To explain this, we introduce some notation and results from Schlichting [11. Let $\mathfrak{M}$ be a collection of "models" and let $D(\mathcal{A})$ be a triangulated category functorially associated to $\mathcal{A} \in \mathfrak{M}$. Here $\mathfrak{M}$ will be the category of complicial bi-Waldhausen categories and $D(\mathcal{A})$ the derived category of $\mathcal{A}$. We loosely speak of the non-connective $K$-theory of $D(\mathcal{A})$ to mean the non-connective $K$-theory of $\mathcal{A}$, as defined in [11.

We say that a sequence $\mathcal{A} \rightarrow \mathcal{B} \rightarrow \mathcal{C}$ is exact in $\mathfrak{M}$ if $D(\mathcal{A}) \rightarrow D(\mathcal{B}) \rightarrow D(\mathcal{C})$ is exact up to direct summands, meaning that $D(\mathcal{A})$ is a thick triangulated subcategory of $D(\mathcal{B})$, that the composite $D(\mathcal{A}) \rightarrow D(\mathcal{C})$ is zero and that the induced functor $D(\mathcal{B}) / D(\mathcal{A}) \rightarrow D(\mathcal{C})$ is fully faithful and cofinal, i.e., is an equivalence after idempotent completion, which means in particular that any object of $D(\mathcal{C})$ is a direct summand of an object of $D(\mathcal{B}) / D(\mathcal{A})$. Given such an exact sequence $\mathcal{A} \rightarrow \mathcal{B} \rightarrow \mathcal{C}$, we have by [11, Thm. 1 , Thm. 6 and $\S 6.5]$ a long exact sequence of $K$-groups :

$\cdots \rightarrow K_{1}(\mathcal{B}) \rightarrow K_{1}(\mathcal{C}) \rightarrow K_{0}(\mathcal{A}) \rightarrow K_{0}(\mathcal{B}) \rightarrow K_{0}(\mathcal{C}) \rightarrow K_{-1}(\mathcal{A}) \rightarrow K_{-1}(\mathcal{B}) \rightarrow \cdots$.

In our situation, since the filtration (6) can be defined on the level of models, and using our abuse of notation $K_{*}(D(\mathcal{A}))=K_{*}(\mathcal{A})$, we get a long exact sequence

$\cdots \rightarrow K_{n}\left(\mathrm{D}_{p-1}\right) \rightarrow K_{n}\left(\mathrm{D}_{p}\right) \rightarrow K_{n}\left(\widetilde{\mathrm{D}_{p} / \mathrm{D}_{p-1}}\right) \rightarrow K_{n-1}\left(\mathrm{D}_{p-1}\right) \rightarrow \cdots \quad(n \in \mathbb{Z})$.

So, we obtain as usual an exact couple and the associated spectral sequence, which looks as follows in cohomological indexing: $E_{1}^{p, q}=K_{-p-q}\left(\mathrm{D}_{-p} \widetilde{\mathrm{D}_{-p-1}}\right)$. This spectral sequence has the desired form by (77) and by agreement of the negative $K$-theory of [11] with Thomason's; see [11, Thm. 5]. Convergence is clear since $E_{1}^{p, q}$ is concentrated in the band $-d \leq p \leq d$ by (3).

Remark 3 . We can replace everywhere $K$-theory by any cohomology theory which associates a long exact sequence to every short sequence of triangulated categories $\mathcal{J} \rightarrow \mathcal{K} \rightarrow \mathcal{L}$ which is exact up to direct summands (see above). This is true in particular for triangular Witt groups tensored with $\mathbb{Z}\left[\frac{1}{2}\right]$ by the localization theorem [1, 6.2 and 6.8] and the cofinality theorem of Hornbostel and Schlichting [7, App. A].

Definition 4. Let $X$ be a (topologically) noetherian scheme of finite Krull dimension $d$. For any $q \in \mathbb{Z}$, we define the $q^{\text {th }}$ augmented weak Gersten complex

$$
\begin{aligned}
0 \rightarrow & K_{q}(X) \rightarrow \bigoplus_{x \in X^{(0)}} K_{q}\left(\mathcal{O}_{X, x}\right) \rightarrow \bigoplus_{x \in X^{(1)}} K_{q-1}\left(\mathcal{O}_{X, x} \text { on }\{x\}\right) \rightarrow \cdots \\
& \cdots \rightarrow \bigoplus_{x \in X^{(p)}} K_{q-p}\left(\mathcal{O}_{X, x} \text { on }\{x\}\right) \rightarrow \cdots \rightarrow \bigoplus_{x \in X^{(d)}} K_{q-d}\left(\mathcal{O}_{X, x} \text { on }\{x\}\right) \rightarrow 0
\end{aligned}
$$


to be the $-q^{\text {th }}$ line of the $E_{1}$-page of our coniveau spectral sequence (1), augmented by the edge homomorphism $K_{q}(X) \rightarrow \bigoplus_{x \in X^{(0)}} K_{q}\left(\mathcal{O}_{X, x}\right)$, which is just localization. We can of course drop the "on $\{x\}$ " when $x \in X^{(0)}$, since there $\{x\}=\operatorname{Spec}\left(\mathcal{O}_{X, x}\right)$. A similar complex exists with dimension instead of codimension and differs from the above in general. We call these complexes weak Gersten complexes because they differ from Gersten complexes for regular schemes in that we cannot replace the local terms by the $K$-groups of the residue fields, since we do not have dévissage in this context.

Remark 5. After Mochizuki [9], one might say that a local noetherian ring $R$ satisfies the generalized Gersten conjecture if the augmented weak Gersten complexes are exact for $X=\operatorname{Spec}(R)$, for all $q \in \mathbb{Z}$. This is equivalent to the vanishing of $K_{n}\left(\mathrm{D}^{p+1}\right) \rightarrow K_{n}\left(\mathrm{D}^{p}\right)$ for all $n \in \mathbb{Z}$ and $p \geq 0$, where the subcategory $\mathrm{D}^{p}:=\left\{E \in \mathrm{D}^{\text {perf }}(X) \mid \operatorname{codim}(\operatorname{supph}(E)) \geq p\right\}$ is again nothing but $\mathrm{D}_{-p}$ in notation (5) applied to the dimension function - codim $_{\text {Krull }}$.

Remark 6. For $q \geq 2$ this conjecture is known to fail in general. For instance, for the local domain $R$ obtained by localization of a cusp at its singularity, say $R=k\left[t^{2}, t^{3}\right]_{\left(t^{2}, t^{3}\right)}$, the (edge) homomorphism $K_{2}(R) \rightarrow K_{2}(k(t))$ is not injective as proved in Dennis and Sherman [6. Appendix], where other examples are provided.

Proposition 7. If $\mathcal{O}_{X, x}$ satisfies the above generalized Gersten conjecture for every $x \in X$, then the $E_{2}$-page of our spectral sequence (11) agrees with that of Thomason (2). Conversely, for $R$ local, if the $E_{2}$-page of our spectral sequence agrees with Thomason's on $X=\operatorname{Spec}(R)$, then the generalized Gersten conjecture holds for $R$.

Proof. The argument is standard. Assume the weak Gersten complex locally exact on $X$. Since the unaugmented complex (i.e., without $K_{q}(X)$ at the beginning) is already a complex of skyscraper Zariski sheaves on $X$, it defines a flasque resolution of the sheafification $\mathcal{K}_{q}$ of the presheaf $U \mapsto K_{q}(U)$. Hence, the homology of this complex, which sits in our $E_{2}$-page, equals the Zariski cohomology of $\mathcal{K}_{q}$. Conversely, for $X=\operatorname{Spec}(R)$ local, suppose the $E_{2}$-page of our spectral sequence consists of Zariski cohomology. Then it vanishes except for $p=0$, which is equivalent to the exactness of the augmented weak Gersten complex.

Remark 8. By Proposition 7 and Remark [6, our coniveau spectral sequence (11) differs from Thomason's (2) in general. As already mentioned, an advantage of our spectral sequence is that it can be non-trivial even for local schemes, where there is no non-trivial cohomology.

We now give an example of a local ring $R$ for which the inclusion $\mathrm{D}^{p+1} \hookrightarrow \mathrm{D}^{p}$ does not even induce the zero map on $K_{0}$ (see Remark 5 ).

Let $k$ be an infinite field and define the ring $R$ as the localization of $k[x, y, z, w]=$ $k[X, Y, Z, W] /\langle X W-Y Z\rangle$ at the point $\mathfrak{m}=\langle x, y, z, w\rangle$. In [5], Dutta, Hochster and McLaughlin provide examples of finite length $R$-modules $M$ which admit a finite projective resolution (that is, $M \in \mathrm{D}_{\{\mathfrak{m}\}}^{\text {perf }}(R)=\mathrm{D}^{3}$ ) but with the property that

$$
\chi(M, R / \mathfrak{p}) \neq 0 \quad(\text { and even } \chi(M, R / \mathfrak{p})<0)
$$

for some $\mathfrak{p} \in \operatorname{Spec}(R)^{(1)}$, for instance for $\mathfrak{p}=\langle x, y\rangle$; see [5, middle of p. 254]. Of course, $\chi\left(M_{1}, M_{2}\right)=\sum(-1)^{i} \operatorname{length}\left(\operatorname{Tor}_{i}^{R}\left(M_{1}, M_{2}\right)\right)$ is Serre's intersection multiplicity. Note that this $\chi\left(M_{1}, M_{2}\right) \in \mathbb{Z}$ is well-defined for any perfect complex 
$M_{1} \in \mathrm{D}^{\text {perf }}(R)=\mathrm{K}^{\mathrm{b}}(R$-free $)$ and any finitely generated $R$-module $M_{2}$ satisfying $\operatorname{supph}\left(M_{1}\right) \cap \operatorname{supp}\left(M_{2}\right) \subset\{\mathfrak{m}\}$. In this case $\operatorname{Tor}_{i}^{R}\left(M_{1}, M_{2}\right)$ simply is the homology in degree $i$ of the (naive) tensor product $M_{1} \otimes M_{2}$; these homology groups are finite length $R$-modules $\operatorname{since} \operatorname{supph}\left(M_{1} \otimes M_{2}\right) \subset \operatorname{supph}\left(M_{1}\right) \cap \operatorname{supp}\left(M_{2}\right) \subset\{\mathfrak{m}\}$.

Proposition 9 (Levine). With the above notation, $[M] \in K_{0}\left(\mathrm{D}^{3}\right)$ does not map to zero under the canonical homomorphism $K_{0}\left(\mathrm{D}^{3}\right) \rightarrow K_{0}\left(\mathrm{D}^{2}\right)$. In particular, this local ring $R$ does not satisfy the generalized Gersten Conjecture of Remark 5.

Proof. For each $a \in k$ consider the prime $\mathfrak{p}_{a}:=\langle x-a z, y-a w\rangle \in \operatorname{Spec}(R)^{(1)}$. Note that for $a \neq b$, we have $\mathfrak{p}_{a}+\mathfrak{p}_{b}=\langle x, y, z, w\rangle=\mathfrak{m}$. Hence, for each $\mathfrak{q} \in \operatorname{Spec}(R)$ with $\mathfrak{q} \neq \mathfrak{m}$, we have at most one $a \in k$ such that $\mathfrak{p}_{a} \subset \mathfrak{q}$. Consider now a closed subset $C \subset \operatorname{Spec}(R)$ such that $\operatorname{codim}(C)=2$. Then $C=V\left(\mathfrak{q}_{1}\right) \cup \ldots \cup V\left(\mathfrak{q}_{n}\right)$ for prime ideals $\mathfrak{q}_{1}, \ldots, \mathfrak{q}_{n} \in \operatorname{Spec}(R)^{(2)}$. By the above considerations, since the field $k$ is infinite, there exists an element $a \in k$ such that $\mathfrak{p}_{a} \not \subset \mathfrak{q}_{i}$ for all $i=1, \ldots, n$. Therefore, $C \cap V\left(\mathfrak{p}_{a}\right)=V\left(\mathfrak{q}_{1}+\mathfrak{p}_{a}\right) \cup \cdots \cup V\left(\mathfrak{q}_{n}+\mathfrak{p}_{a}\right)=\{\mathfrak{m}\}$.

Let us assume $a b$ absurdo that $[M] \mapsto 0 \in K_{0}\left(\mathrm{D}^{2}\right)$. For $C \subset \operatorname{Spec}(R)$, recall the standard notation $\mathrm{D}_{C}^{\text {perf }}(R):=\left\{E \in \mathrm{D}^{\text {perf }}(R) \mid \operatorname{supph}(E) \subset C\right\}$. Since we have

$$
K_{0}\left(\mathrm{D}^{2}\right)=\underset{\substack{C \subset \operatorname{coc}(R), \text { closed } \\ \text { with } \operatorname{codim}(C) \geq 2}}{\operatorname{colim}} K_{0}\left(\mathrm{D}_{C}^{\text {perf }}(R)\right),
$$

we can assume that $[M] \mapsto 0$ in $K_{0}\left(D_{C}^{\text {perf }}(R)\right)$ for some closed subset $C \subset \operatorname{Spec}(R)$ with $\operatorname{codim}(C) \geq 2$, that is, $\operatorname{codim}(C)=2$ or $C=\{\mathfrak{m}\}$. In any case, by the first part of the proof, there exists $a \in k$ such that $C \cap V\left(\mathfrak{p}_{a}\right)=\{\mathfrak{m}\}$. Let us fix such a $C \subset \operatorname{Spec}(R)$ and such an $a \in k$.

In this situation, the intersection multiplicity $\chi\left(E, R / \mathfrak{p}_{a}\right) \in \mathbb{Z}$ is defined for any $E \in \mathrm{D}_{C}^{\text {perf }}(R)$. Since it is additive on distinguished triangles in $\mathrm{D}_{C}^{\text {perf }}(R)$, the expression $\chi\left(E, R / \mathfrak{p}_{a}\right)$ only depends on the class of $E$ in $K_{0}\left(\mathrm{D}_{C}^{\text {perf }}(R)\right)$. Therefore, our assumption $[M]=0$ in $K_{0}\left(\mathrm{D}_{C}^{\text {perf }}(R)\right)$ implies

$$
\chi\left(M, R / \mathfrak{p}_{a}\right)=0 .
$$

Similarly, since $M \in \mathrm{D}_{\{\mathfrak{m}\}}^{\text {perf }}(R)$, the number $\chi\left(M, M_{2}\right)$ is defined for any finitely generated $R$-module $M_{2}$ and, as before, induces a well-defined homomorphism $\chi(M,-)$ on $K_{0}\left(R\right.$-mod) $=G_{0}(R)$. To get a contradiction between (8) and (9), hence to finish the proof, it now suffices to show that $\left[R / \mathfrak{p}_{a}\right]=[R / \mathfrak{p}]$ in $G_{0}(R)$ for all $a \in k$. To see this, it is enough to check that the following sequence is exact:

$$
0 \rightarrow \operatorname{Ker}(\beta) \rightarrow R^{2} \stackrel{\beta}{\longrightarrow} R^{2} \stackrel{\alpha_{a}}{\longrightarrow} R \rightarrow R / \mathfrak{p}_{a} \rightarrow 0,
$$

where $\alpha_{a}=\left(\begin{array}{ll}x-a z & y-a w\end{array}\right)$ and $\beta=\left(\begin{array}{cc}w & -y \\ -z & x\end{array}\right)$, for this will imply that $\left[R / \mathfrak{p}_{a}\right]=$ $[R]-[\operatorname{Ker}(\beta)]$ in $G_{0}(R)$ and the latter is independent of $a \in k$. In fact, only exactness at the middle $R^{2}$ requires proof, and this can be given in the spirit of [5, Lemma 2.2]. Since localization is exact, we prove exactness of (10) with $R$ replaced by $R=k[x, y, z, w]=k[X, Y, Z, W] /\langle X W-Y Z\rangle$. Let $\left(\begin{array}{l}u \\ v\end{array}\right) \in R^{2}$ in the kernel of $\alpha_{a}$. Let $U, V \in k[X, Y, Z, W]$ such that $U \mapsto u$ and $V \mapsto v$ in $R$. Then $\alpha_{a} \cdot\left(\begin{array}{l}u \\ v\end{array}\right)=0$ reads

$$
(X-a Z) U+(Y-a W) V=(X W-Y Z) G
$$

for some $G \in k[X, Y, Z, W]$. A direct computation from the above gives

$$
(X-a Z)(U-W G)=-(Y-a W)(V+Z G) .
$$


This implies that $(Y-a W) \mid(U-W G)$, i.e., $U=W G+(Y-a W) H$ for some $H \in k[X, Y, Z, W]$. Replacing $U$ in the last equation and canceling by $(Y-a W)$ gives $V=-Z G-(X-a Z) H$. Finally, defining $g$ and $h$ in $R$ to be the classes of $G$ and $H$ respectively, we obtain $\left(\begin{array}{l}u \\ v\end{array}\right)=\left(\begin{array}{cc}w & -y \\ -z & x\end{array}\right) \cdot\left(\begin{array}{c}g-a h \\ -h\end{array}\right)$, as was to be shown.

Remark 10. One can visualize the above proof by tracking the following objects:

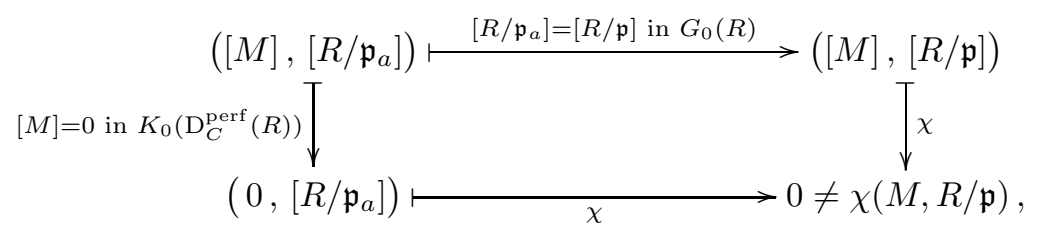

in the commutative diagram

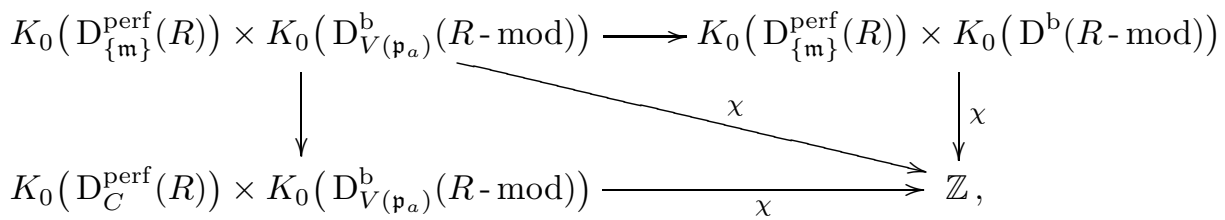

whose lower homomorphism $\chi$ is well-defined because of the construction of $a \in k$ such that $C \cap V\left(\mathfrak{p}_{a}\right)=\{\mathfrak{m}\}$, made at the very beginning of the proof.

Finally, we discuss a condition for (1) to be a fourth quadrant spectral sequence.

Conjecture 11 (Weibel [14, 2.9]). For $X$ a noetherian scheme of Krull dimension $d$, we have $K_{m}(X)=0$ for $m<-d$.

Very recently, this conjecture has been proved for $X$ essentially of finite type over a field of characteristic zero by Cortiñas-Haesemeyer-Schlichting-Weibel [4]. As already observed by Vorst [13, Cor. 1.9 (iii)], at least in the reduced affine case, we now show that it suffices to prove Conjecture 11 for local rings. (This can also be proved with Thomason's hypercohomology spectral sequence.)

Proposition 12. Let $X$ be a noetherian scheme of Krull dimension d. Then the following are equivalent:

(i) Conjecture 11 holds for the local ring $\mathcal{O}_{X, x}$ for every $x \in X$.

(ii) For all $p \geq 0$, all $x \in X^{(p)}$ and all $q>0$ we have $K_{-p-q}\left(\mathcal{O}_{X, x}\right.$ on $\left.\{x\}\right)=0$. Moreover, when (i) and (ii) hold true, then $X$ satisfies Conjecture 11 as well.

Proof. Of course $E_{1}^{p, q}=0$ except for $0 \leq p \leq d$ since $X^{(p)}$ is empty for other $p \in \mathbb{Z}$.

Claim. Suppose that $K_{-p-q}\left(\mathcal{O}_{X, x}\right.$ on $\left.\{x\}\right)=0$ for all $0 \leq p \leq d-1$, all $x \in X^{(p)}$ and for all $q>0$. Then for $n>d$, we have $K_{-n}(X) \cong \bigoplus_{x \in X^{(d)}} K_{-n}\left(\mathcal{O}_{X, x}\right.$ on $\left.\{x\}\right)$.

The hypothesis of the claim means that in our spectral sequence (1), we have $E_{1}^{p, q}=0$ for $p \neq d$ and $q>0$. So, for $q>0$, we have $E_{1}^{p, q}=E_{\infty}^{p, q}$ and this group vanishes except for $p=d$ where it gives the above direct sum. Hence the claim.

Assume (i) and let us prove (ii) by induction on $p \geq 0$. For $p=0$, we have of course $K_{*}\left(\mathcal{O}_{X, x}\right.$ on $\left.\{x\}\right)=K_{*}\left(\mathcal{O}_{X, x}\right)$, so (ii) is true by assumption (i). For $p \geq 1$ and $x \in X^{(p)}$, apply the claim to the scheme $\operatorname{Spec}\left(\mathcal{O}_{X, x}\right)$. The hypothesis of the claim holds by the induction hypothesis. Then, for $q>0$, the conclusion of the 
claim applied to $n=p+q$ gives $K_{-p-q}\left(\mathcal{O}_{X, x}\right)=K_{-p-q}\left(\mathcal{O}_{X, x}\right.$ on $\left.\{x\}\right)$ and the former vanishes by (i). This proves (ii).

Conversely, assume (ii). Then the first page of our spectral sequence (11) vanishes for $q>0$. Since it is concentrated in $0 \leq p \leq d$ and converges to $K_{*}(X)$, we get Conjecture 11 for $X$. This already shows the "moreover part" of the statement. This implication '(ii) $\Rightarrow$ Conj.[11' applied to $X=\operatorname{Spec}\left(\mathcal{O}_{X, x}\right)$ also proves (ii) $\Rightarrow(\mathrm{i})$, since (ii) is a local property.

Corollary 13. Conjecture 11 is equivalent to: For every noetherian local ring $(R, \mathfrak{m})$ of Krull dimension $d$ and all $n>d$, we have $K_{-n}(\operatorname{Spec}(R)$ on $\{\mathfrak{m}\})=0$. This is also equivalent to our coniveau spectral sequence (11) being concentrated in the fourth quadrant ( $p \geq 0$ and $q \leq 0$ ) for every finite dimensional noetherian scheme $X$.

\section{ACKNOWLEDGMENTS}

The author thanks Henri Gillet for useful discussions, Marc Levine for kindly providing Proposition 9, Marco Schlichting for precious comments and for the reference to [6], and Chuck Weibel for several references, among them [13. Finally, thanks to the referee for remarks and references.

\section{REFERENCES}

[1] P. Balmer. Triangular Witt groups. I. The 12-term localization exact sequence. K-Theory, 19(4):311-363, 2000. MR.1763933 (2002h:19002)

[2] P. Balmer. Supports and filtrations in algebraic geometry and modular representation theory. Amer. J. Math., 129(5):1227-1250, 2007. MR.2354319

[3] P. Balmer and M. Schlichting. Idempotent completion of triangulated categories. J. Algebra, 236(2):819-834, 2001. MR.1813503 (2002a:18013)

[4] G. Cortiñas, C. Haesemeyer, M. Schlichting, and C. Weibel. Cyclic homology, cdh-cohomology and negative $K$-theory, Ann. of Math., to appear.

[5] S. P. Dutta, M. Hochster, and J. E. McLaughlin. Modules of finite projective dimension with negative intersection multiplicities. Invent. Math., 79(2):253-291, 1985. MR778127 (86h:13023)

[6] S. C. Geller. A note on injectivity of lower $K$-groups for integral domains. In Applications of algebraic K-theory to algebraic geometry and number theory, Parts I, II (Boulder, Colo., 1983), volume 55 of Contemp. Math., pages 437-447, Amer. Math. Soc., Providence, RI, 1986. With an appendix by R. Keith Dennis and Clayton C. Sherman. MR862647 (87k:18013)

[7] J. Hornbostel and M. Schlichting. Localization in Hermitian $K$-theory of rings. J. London Math. Soc. (2), 70(1):77-124, 2004. MR2064753 (2005b:19007)

[8] M. Levine. Localization on singular varieties. Invent. Math., 91(3):423-464, 1988. MR928491 (89c:14015a)

[9] S. Mochizuki. Gersten's conjecture for $K_{0}$-groups. www math.uiuc.edu/K-theory/0842, 2007.

[10] D. Quillen. Higher algebraic K-theory. I. Algebraic K-theory, I: Higher K-theories (Proc. Conf., Battelle Memorial Inst., Seattle, Wash., 1972), pages 85-147, Lecture Notes in Math., Vol. 341, Springer, Berlin, 1973. MR0338129 (49:2895)

[11] M. Schlichting. Negative $K$-theory of derived categories. Math. Z., 253(1):97-134, 2006. MR2206639 (2006i:19003)

[12] R. W. Thomason and T. Trobaugh. Higher algebraic $K$-theory of schemes and of derived categories. The Grothendieck Festschrift, Vol. III, volume 88 of Progr. Math., pages 247-435. Birkhäuser, Boston, MA, 1990. MR1106918 (92f:19001)

[13] T. Vorst. Localization of the K-theory of polynomial extensions. Math. Ann., 244(1):33-53, 1979. With an appendix by Wilberd van der Kallen. MR 550060 (80k:18016)

[14] C. A. Weibel. K-theory and analytic isomorphisms. Invent. Math., 61(2):177-197, 1980. MR.590161 (83b:13011) 
[15] C. A. Weibel. Negative $K$-theory of varieties with isolated singularities. In Proceedings of the Luminy conference on algebraic K-theory (Luminy, 1983), volume 34, pages 331-342, 1984. MR.772067 (86d:14015)

[16] C. A. Weibel. A Brown-Gersten spectral sequence for the $K$-theory of varieties with isolated singularities. Adv. in Math., 73(2):192-203, 1989. MR987274 (90j:14012)

[17] C. A. Weibel. A Quillen-type spectral sequence for the $K$-theory of varieties with isolated singularities. K-Theory, 3(3):261-270, 1989. MR.1040402(91g:14009)

Department of Mathematics, Box 951555, University of California, Los Angeles, CALIFORNia 90095-1555

E-mail address: balmer@math.ucla.edu

URL: http://www. math.ucla.edu/ balmer 